This item was submitted to Loughborough's Research Repository by the author.

Items in Figshare are protected by copyright, with all rights reserved, unless otherwise indicated.

\title{
Oblique incidence performance of a novel frequency selective surface absorber
}

PLEASE CITE THE PUBLISHED VERSION

PUBLISHER

(C) Institute of Electrical and Electronics Engineers (IEEE)

LICENCE

CC BY-NC-ND 4.0

REPOSITORY RECORD

Kiani, Ghaffer I., Kenneth L. Ford, Karu P. Esselle, Andrew R. Weily, and C.J. Panagamuwa. 2019. "Oblique Incidence Performance of a Novel Frequency Selective Surface Absorber". figshare.

https://hdl.handle.net/2134/3092. 
This item was submitted to Loughborough's Institutional Repository by the author and is made available under the following Creative Commons Licence conditions.

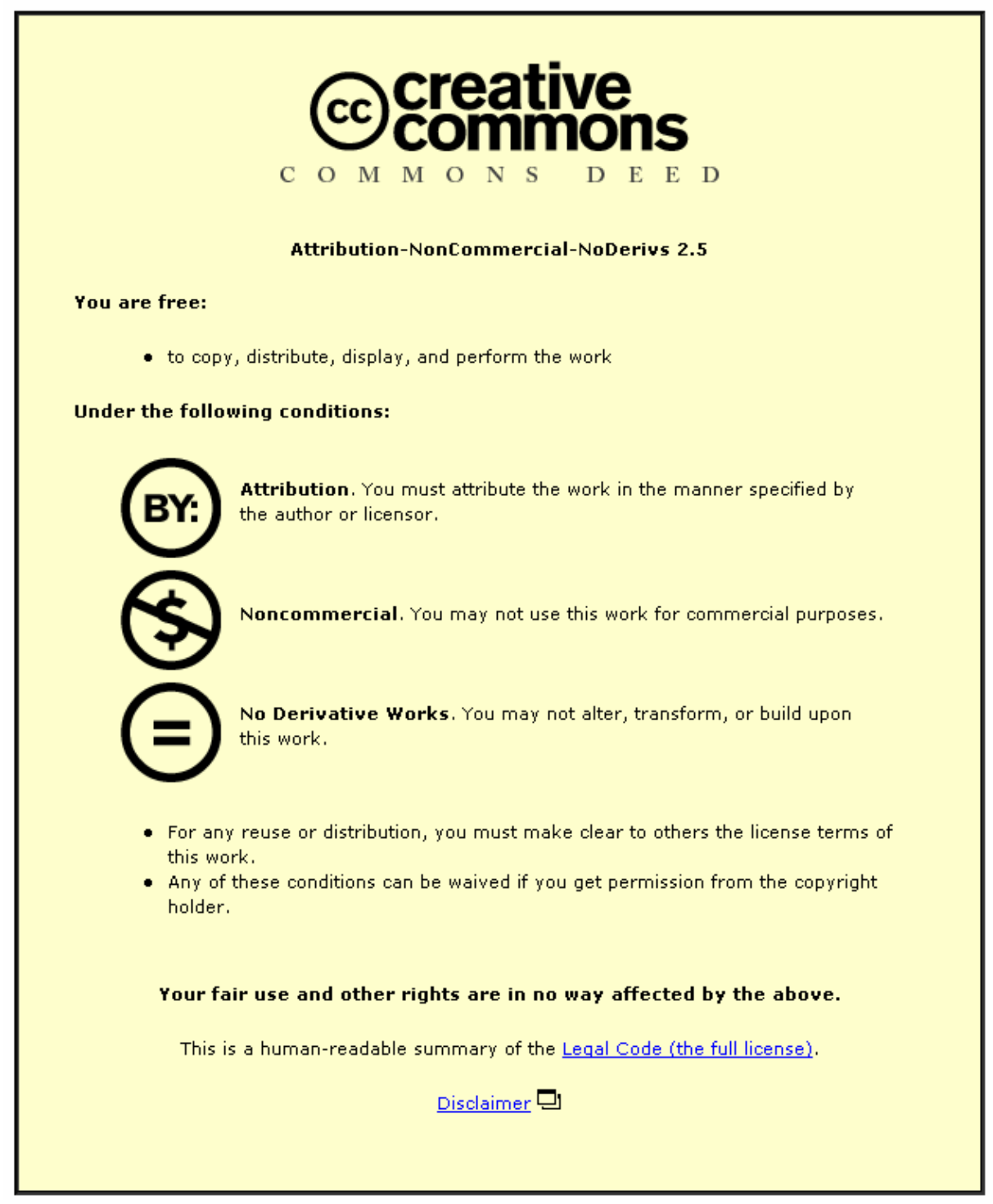

For the full text of this licence, please go to: http://creativecommons.org/licenses/by-nc-nd/2.5/ 
[6] W. J. Byun, J. W. Yu, and N. H. Myung, "TM scattering from hollow and dielectric-filled semielliptic channels with arbitrary eccentricity in a perfectly conducting plane," IEEE Microw. Theory Tech., vol. 46, no. 9, pp. 1336-1339, Sep. 1998.

[7] V. P. Chumachenko, E. Karacuha, and M. Dumanli, "TM-scattering from a multiangular groove in a ground plane," J. Electromagn. Waves Applicat., vol. 14, no. 3, pp. 329-347, 2000.

[8] H. A. Ragheb, "Electromagnetic scattering from a coaxial dielectric circular cylinder loading a semicircular gap in a ground plane," IEEE Trans. Microw. Theory Tech., vol. 43, no. 6, pp. 1303-1309, Jun. 1995.

[9] G. N. Watson, A Treatise on the Theory of Bessel Functions, 2nd ed. Cambridge, U.K.: Cambridge Univ. Press, 1958.

\section{Oblique Incidence Performance of a Novel Frequency Selective Surface Absorber}

\author{
Ghaffer I. Kiani, Kenneth L. Ford, Karu P. Esselle, \\ Andrew R. Weily, and Chinthana J. Panagamuwa
}

\begin{abstract}
Oblique incidence performance of a novel two-layer absorb/ transmit frequency selective surface (FSS) is investigated. The FSS has good frequency stability for both horizontally and vertically polarized waves incident normally or at oblique angles. Due to its transmission for 900/1800/ $1900 \mathrm{MHz}$ mobile bands and good absorption for $5 \mathrm{GHz}$ waves, it has the potential as a security wall or isolator for $5 \mathrm{GHz}$ WLAN systems. The absorption in the stop band helps reduce additional WLAN multipath fading caused by conventional reflecting FSS designs. The first layer of the FSS consists of conventional conducting cross dipoles having a circular aperture in the centre, while the second layer uses resistive cross dipoles. Moreover, the conducting cross dipoles have been sandwiched between two dielectric sheets to achieve a stable response for different angles of incidence. The periodicity of both FSS layers is the same while the distance between the two layers is reduced to one eighth of the free-space wavelength. This reduction leads to a more compact design compared to the conventional Salisbury screen, while still achieving acceptable absorption in the stopband. Both theoretical and experimental results are presented to confirm the performance of the absorb/transmit FSS.
\end{abstract}

Index Terms-Absorber, delay spread, fading, frequency selective surface (FSS), multilayers, multipath, oblique incidence, security, two-layers, wireless networks, WLAN.

\section{INTRODUCTION}

Frequency selective surfaces (FSSs) have been developed for use as spatial filters of microwaves and millimeter waves [1]. They have been considered for applications such as wireless security, radomes, antennas and telecommunications [2]. Common FSSs, which are reflect/transmit type, can be incorporated in the walls of buildings to provide wireless local area network (WLAN) security. They block WLAN

Manuscript received March 15, 2007; revised May 11, 2007. This work was supported in part by Macqaurie University, Sydney, Australia, and in part by the University of Sheffield.

G. I. Kiani and K. P. Esselle are with the Department of Electronic Engineering, Macquarie University, Sydney, New South Wales 2109, Australia (e-mail: gkiani@ics.mq.edu.au).

K. L. Ford is with the Department of Electronic and Electrical Engineering, University of Sheffield, Sheffield S1 3JD, U.K.

A. R. Weily is with CSIRO ICT Centre, Epping, New South Wales 1710, Australia.

C. J. Panagamuwa is with the Department of Electronic and Electrical Engineering, Loughborough University, Loughborough LE11 3TU, U.K.

Digital Object Identifier 10.1109/TAP.2007.905980 signals while passing cellular phone signals [3] but give rise to heavy stopband reflections from the FSS surface causing additional delay spread and multipath fading in WLAN systems. Research has recently been undertaken to use lossy FSS as absorbers [4]-[6]. The main challenges in these designs are: a) to achieve stable response for oblique incidence for both polarizations (TE and TM); and b) to reduce the distance between the FSS and resistive sheets to achieve a compact design [4] without compromising absorption performance. Moreover, research has also been carried out to achieve stable frequency response for oblique angles from circuit analog absorbers at both parallel and perpendicular polarizations [7].

In this communication we present a novel absorb/transmit FSS designed to provide security and/or isolation for $5 \mathrm{GHz}$ systems. It passes cellular phone signals and blocks $5 \mathrm{GHz}$ signals by absorbing, as opposed to reflecting. The FSS is a two-layer structure, with conducting cross dipoles on one layer and resistive cross dipoles on the second layer. The advantages of the design are: 1) it provides absorption in 5 $\mathrm{GHz}$ band; 2) it maintains transmission of $900 / 1800 / 1900 \mathrm{MHz}$ mobile phone bands; 3) the frequency of maximum attenuation remains relatively stable for different incident angles; and 4) it is more compact than conventional designs making it more practical for commercial use. These properties give the absorb/transmit FSS a clear advantage over conventional Salisbury and Jaumann absorbers, which typically can provide good absorption in the stopband but have poor out of band transmission.

In this design the frequency stability of both polarizations has been achieved by three techniques. Firstly, the inter-element spacing between cross dipoles has been reduced [1]. This improves its frequency response for oblique angles and has added considerable stability for both polarizations. Secondly, a small circular aperture has been etched at the centre of the conducting cross dipole to change its surface impedance. For a thin surface, the total electric field on the surface is equal to the product of the surface impedance and the surface current density, which can be represented as

$$
\vec{E}^{\text {inc }}+\vec{E}^{\text {scat }}=Z_{s} \vec{J}_{s}
$$

where $Z_{s}$ is the surface impedance. The two limiting cases for this equation occur when $Z_{s}=0$ or when $Z_{s}$ approaches infinity. The boundary condition enforced by this equation is a PEC when $Z_{s}=0$ (short-circuit). On the other hand if $Z_{s}$ approaches infinity, the surface currents are forced to zero and hence no energy is scattered from the surface (open-circuit). In our cross dipole case, the open-circuit condition is approximated because removing patch from the center of the cross dipole increases the surface impedance [8]. The additional inductance due to narrow metal around the hole also contributes to tuning the FSS elements to resonate at $5 \mathrm{GHz}$. Thirdly, the conducting cross dipoles have been sandwiched between two dielectric layers to provide extra frequency stability and power handling [1]. There is no major improvement in the stability or the absorption of FSS absorber when the resistive cross dipole is sandwiched within two FR4 sheets.

\section{CONFIGURATION}

The unit cell configuration of the absorb/transmit FSS is shown in Figs. 1 and 2. The $5 \mathrm{GHz}$ stopband filter characteristics are achieved by incorporating an array of conducting cross dipoles on one side of one of the FR4 sheets. Another sheet of FR4 with the same dielectric constant and thickness is placed on the open side of the array of conducting dipoles. This sandwiched configuration is shown in Fig. 1. The absorption around $5 \mathrm{GHz}$ is achieved by incorporating a layer of 


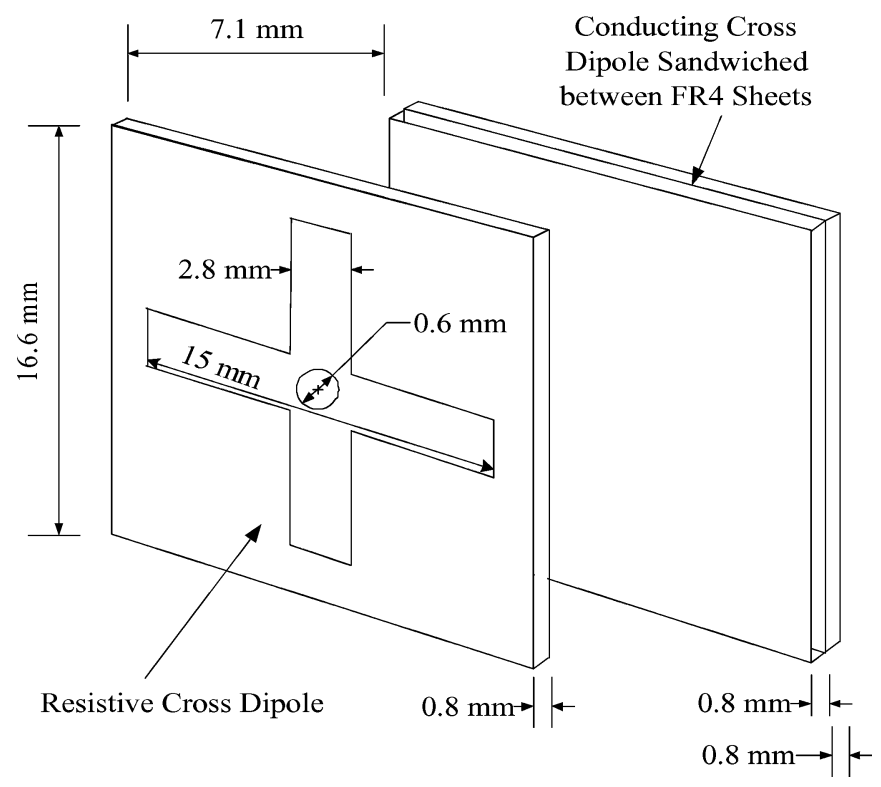

Fig. 1. Unit cell of the dual-layer absorb/transmit FSS surface with conducting cross dipoles sandwiched between two FR4 sheets.

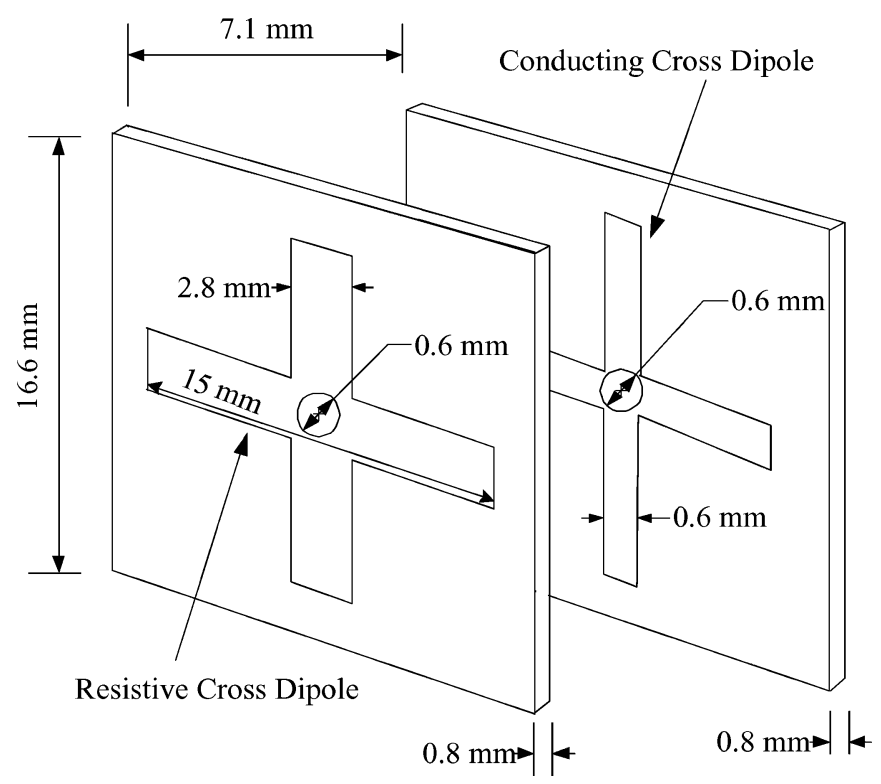

Fig. 2. Unit cell of the dual-layer absorb/transmit FSS surface. The front FR4 sheet of the conducting cross dipole has been removed to show its structure more clearly.

resistive cross dipoles. This two-layer combination operates as an absorb/transmit filter, as follows. The conducting layer behaves as a conventional reflect/transmit filter, reflecting $5 \mathrm{GHz}$ waves while transmitting mobile phone signals. The "matched" resistive layer absorbs the 5 $\mathrm{GHz}$ waves reflected by the conducting layer. The width of the resistive cross dipoles $(2.8 \mathrm{~mm})$ is larger than the conducting cross dipoles $(0.6$ $\mathrm{mm}$ ) to obtain good absorption in the stopband. A conjugate matching for resistive cross dipoles is not attempted here since the absorption is comparatively less and the resistive sheets with low resistance per square may not be readily available. A circular aperture with a diameter of $0.6 \mathrm{~mm}$ has been etched at the center of both the conducting and resistive cross dipoles. This aperture makes the design more stable for polarizations at oblique angles of incidence. Each FR4 sheet is $0.8 \mathrm{~mm}$ thick, and the surface resistance of the resistive dipoles is chosen as 50 $\Omega$ per square, as surfaces with these parameters are readily available.

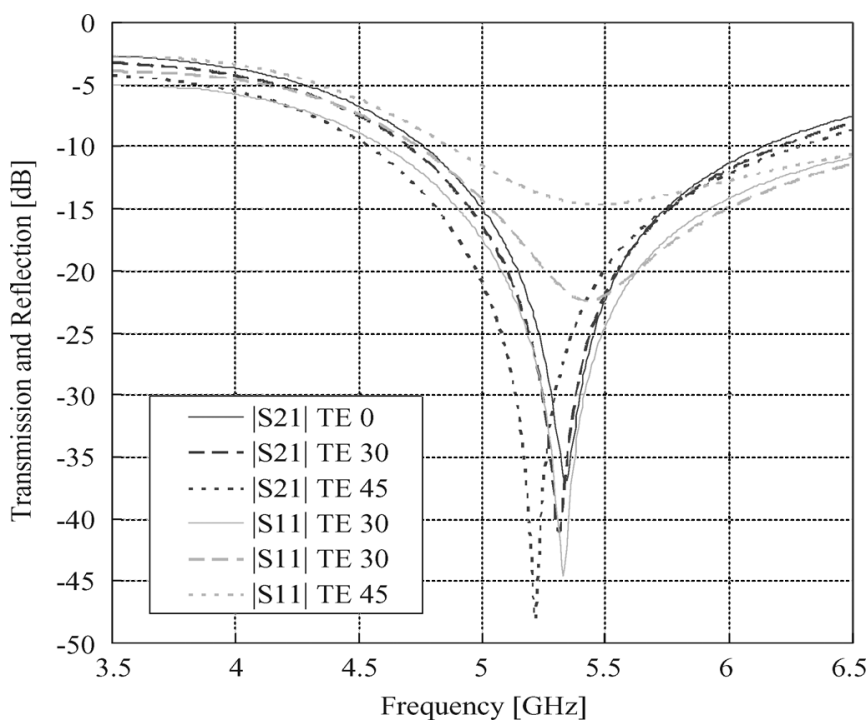

Fig. 3. Theoretical transmission and reflection of the dual-layer absorb/transmit FSS at oblique angles, for perpendicular polarization (TE).

The stability of the conducting cross dipole FSS has been enhanced significantly by reducing inter-element spacing, etching a hole in the center of the dipole elements and by sandwiching the dipoles between two FR4 sheets. The resistive cross dipole concept follows the principle of the conventional Salisbury screen where a uniform resistive sheet is employed for wave absorption. However, unlike in the Salisbury screen, our resistive layer is also a periodic FSS. Its pattern is matched to the conducting layer in periodicity and shape in order to absorb $5 \mathrm{GHz}$ signals reflected by the conduction layer while passing mobile phone signals. The design has a stable frequency of maximum absorption as well as stable absorption response for both parallel and perpendicular polarizations at oblique angles ranging from $0-45$ degrees. The reflection measurements presented in Section III have been carried out in an anechoic chamber at University of Sheffield, U.K., designed to measure oblique angle performance for both polarizations. The transmission measurements have been carried out in a testing facility in Loughborough University, U.K. This facility is also able to measure the transmission coefficient for both polarizations.

\section{THEORETICAL AND EXPERIMENTAL RESULTS}

The theoretical results of absorb/transmit FSS, for perpendicular polarization (TE), are shown in Fig. 3, while the measured results are depicted in Fig. 4.

In Fig. 3, for 0, 30, and 45 degree angles of incidence, the resonance frequencies are 5.32,5.31, and $5.22 \mathrm{GHz}$, the transmission coefficients are $-44.6,-41$, and $-48.1 \mathrm{~dB}$, and the reflection coefficients at the resonance are $-37.2,-21.4$, and $-13.6 \mathrm{~dB}$, respectively. The $-10 \mathrm{~dB}$ stopband bandwidths for 0,30 and 45 degree incident angles are 1.54, 1.54 , and $1.75 \mathrm{GHz}$, respectively.

In Fig. 4, the measured results of absorb/transmit FSS at oblique angles are presented for perpendicular polarization (TE). Once again a higher resonance frequency is observed due to air gap [1, Fig. E.2.] and possibly a lower dielectric constant of FR4 used for the fabrication. For 0,30 , and 45 degree angles of incidence, the resonance frequencies are $5.66,5.67$, and $5.68 \mathrm{GHz}$, the transmission coefficients are -25.1 , -23.6 , and $-20.9 \mathrm{~dB}$ and the reflection coefficients are $-20.6,-25.2$, and $-15.3 \mathrm{~dB}$, respectively. The $-10 \mathrm{~dB}$ stopband bandwidths for 0 , 30 , and 45 degree incident angles are 1.61, 1.66, and $1.85 \mathrm{GHz}$, respectively. As predicted in theoretical results, the frequency of maximum attenuation is very stable with respect to the angle of incidence. The 


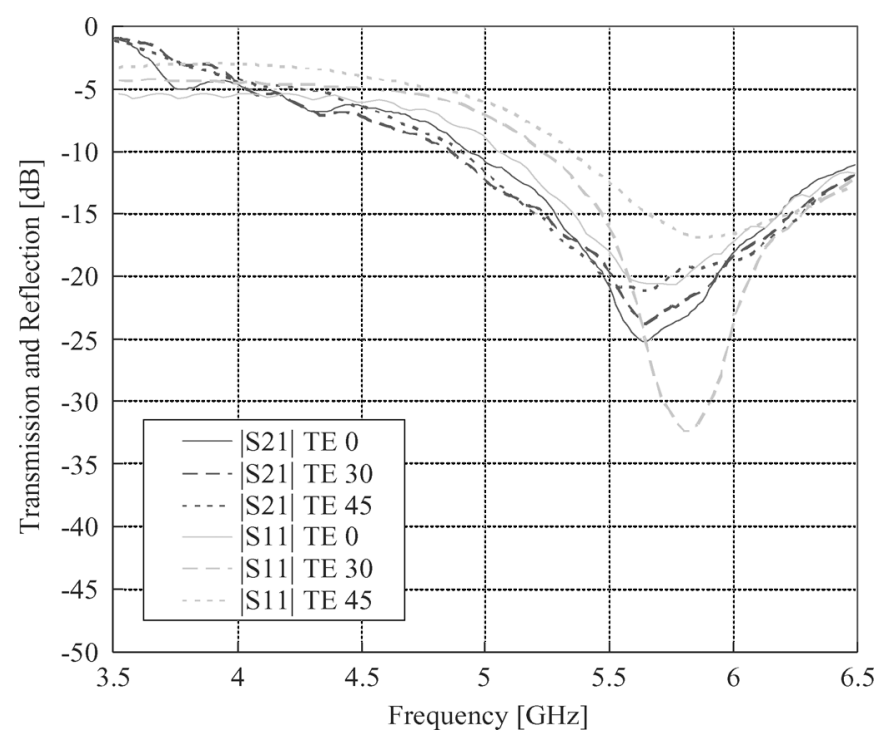

Fig. 4. Measured transmission and reflection of the dual-layer absorb/transmit FSS at oblique angles, for perpendicular polarization (TE).

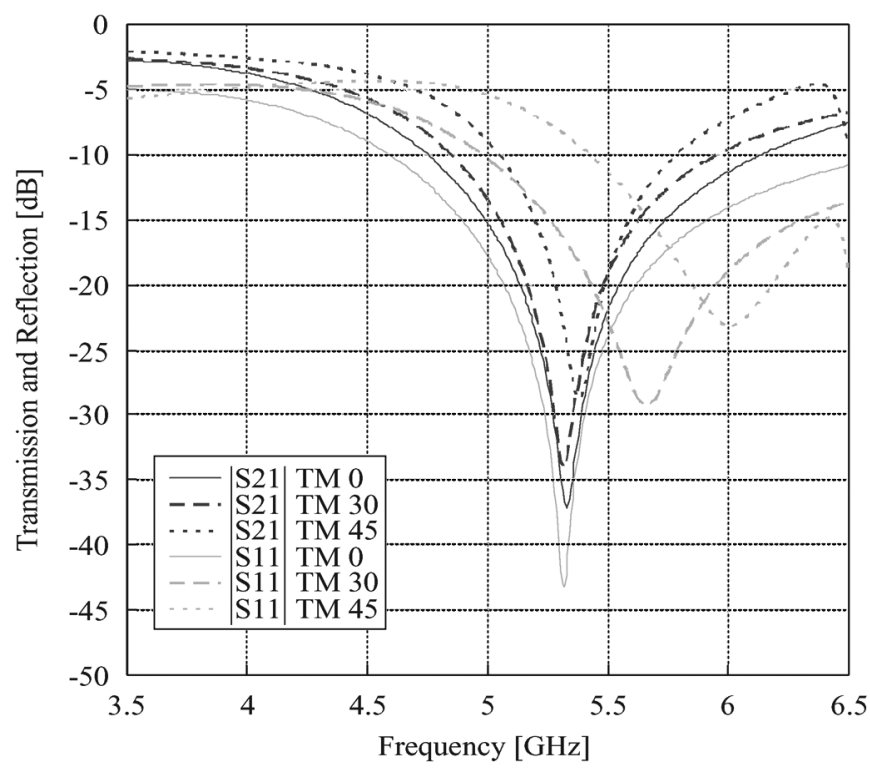

Fig. 5. Theoretical transmission and reflection of the dual-layer absorb/transmit FSS, at oblique angles, for parallel polarization (TM).

variation of absorption and stopband width agree with the trend predicted in theoretical results.

Fig. 5 presents the oblique angle theoretical data of the absorb/transmit FSS for parallel polarized (TM) incident waves. For 0, 30 , and 45 degree angles of incidence, the resonance frequencies are $5.32,5.32$, and $5.38 \mathrm{GHz}$, transmission coefficients are $-43.2,-33.7$, and $-28.6 \mathrm{~dB}$, and the reflection coefficients are $-36.7,-16.9$, and $-9.4 \mathrm{~dB}$, respectively. The $-10 \mathrm{~dB}$ stopband bandwidth for 0,30 , and 45 degree incident angles are $1.88,1.12$, and $0.75 \mathrm{GHz}$, respectively. There is a decrease in the bandwidth with the increase of the angle of incidence. However good frequency stability and absorption characteristics have been achieved in this case as well.

In Fig. 6, the measured data at oblique angles for parallel polarization (TM) are presented. For 0,30 , and 45 degree angles of incidence, the resonance frequencies are $5.7,5.8$, and $5.9 \mathrm{GHz}$, the transmission coefficients are $-30.8,-21.5$, and $-17.8 \mathrm{~dB}$, the reflection coefficients

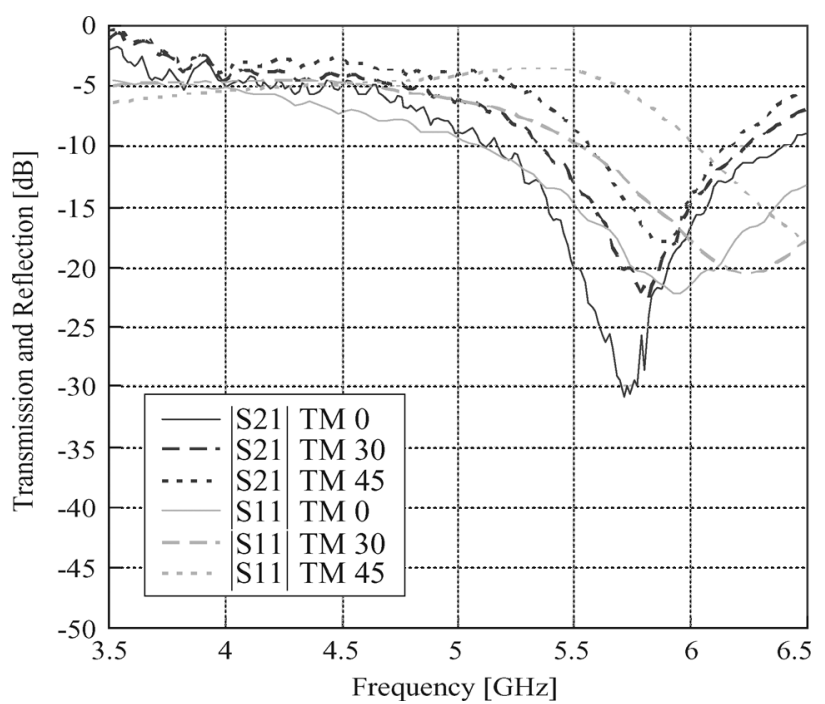

Fig. 6. Measured transmission and reflection of the dual-layer absorb/transmit FSS, at oblique angles, for parallel polarization (TM).

are $-18.8,-14.5$, and -7.7 , and $-10 \mathrm{~dB}$ stopband bandwidths are $1.3,0.9$, and $0.6 \mathrm{GHz}$, respectively. It is interesting to note that the frequency of maximum attenuation does not align with the frequency of minimum reflection, for TM incidence. As a result, the reflection coefficient of the frequency of maximum attenuation is not small as in the TE incidence; nevertheless it is less than $-7.7 \mathrm{~dB}$ for the range of incident angles considered.

\section{DISCUSSION}

Some noise in the transmission measurements was observed for oblique angles of incidence due to the small size of the FSS prototype. The transmission tests were carried out in an anechoic chamber in which the FSS prototype was fitted in a window on a rotatable cardboard sheet. This cardboard sheet was covered with absorber material. The horn antennas were kept on both sides of the cardboard. Therefore, for large angles of incidence measurements, the small transmission window was slightly shadowed by the surrounding absorbers, leading to additional noise in the measured data. Therefore, for the absorb/transmit FSS measured data, averaging has been applied for $45^{\circ}$ to smooth the transmission curves. As it can be noticed that the measured resonant frequencies some times differ from theoretical resonance frequencies. This was due to: a) the air gap between the two dielectric sheets sandwiching the conducting cross dipoles [1, Fig. E.2.]; b) a sag in both layers of absorbers due to their smaller thickness, causing poor mechanical strength; and c) the use of $7.1 \mathrm{~mm}$ spacers only at the edges of the conducting and resistive FSS layers causing nonuniform spacing between the sides and the center.

\section{CONCLUSION}

The performance of a dual-layer cross-dipole absorb/transmit FSS has been demonstrated through both theory and experiments. The prototype showed a stable frequency of maximum attenuation for both polarizations at oblique angles up to 45 degrees. It attenuates $5 \mathrm{GHz}$ waves very well, while providing a useful reduction in their reflection bands, over the range of incident angles and polarizations considered. This absorb/transmit FSS may be adopted for WLAN security in office environments. It provides isolation by absorbing WLAN signals instead of reflecting them. The additional multipath channels and the delay spread that would be created by a reflecting FSS are avoided by the absorb/transmit FSS and hence better signal quality can be achieved. 
Good frequency stability of the design ensures that the FSS will absorb signals over a wide range of incidence angles, not only those incident at normal. In contrast to traditional frequency absorbers such as the Salisbury screen, the absorb/transmit FSS transmits 900/1800/1900 mobile/cellular phone bands to maintain important emergency and personal communication services.

The distance between the two FSS layers is of the order of approximately $1 / 8$ of the free space wavelength, which makes it half as thick as conventional absorbers which have a standard $\lambda / 4$ distance between the layers. Due to the added advantage of compact size, a third FSS layer similar to the resistive FSS layer can be placed on the other side of the conducting FSS layer at a distance of approximately $\lambda / 8$ to create a two way absorber. By adding this second resistive FSS layer, the total distance between the edges of FSS absorber will still remain close to $\lambda / 4$ as in the case of other conventional absorbers. This triple-layer absorber can be incorporated in a wall of a building for advanced wireless security in such a way that its bi-directional absorbing characteristics can mislead an intruder. The intruder would not be able to detect the presence of physical wireless security from outside the building using a low-power microwave transmitter as any test signal sent by the intruder would be absorbed by the FSS absorber in the walls. The intruder would assume full transmission of the test signal through the wall and infer there is no physical barrier.

The absorb/transmit FSS can also help WLAN providers to stop people accessing the service outside of their premises (e.g., restaurants). Moreover, it can divide a large WLAN service area into several small areas thereby increasing frequency reuse and the total number of users the WLAN can support.

\section{ACKNOWLEDGMENT}

The authors thank Prof. R. Langley, Department of Electrical and Electronics Engineering, University of Sheffield for his invitation to carry out collaborative work on FSS. They would also like to extend their gratitude to Prof. Y. Vardaxoglou, Loughborough University, for providing lab facilities for transmission measurement of the absorb/ transmit FSS.

\section{REFERENCES}

[1] B. A. Munk, Frequency Selective Surfaces: Theory and Design. New York: Wiley, 2000

[2] C. Mias, C. Tsakonas, and C. Oswald, "An investigation into the feasibility of designing frequency selective windows employing periodic structures," Radiocommunications Agency Final Rep. The Nottingham Trent Univ. Nottingham, U.K., Ref. AY3922, 2001.

[3] E. A. Parker et al., Application of FSS structures to selectively control the propagation of signals into and out of buildings Prepared by ERA Technology Ltd. and its team for the Ofcom under Contract No. AY4464.

[4] A. Itou, O. Hashimoto, H. Yokokawa, and K. Sumi, "A fundamental study of a thin wave absorber using FSS technology," Electron. Commun. Jpn, vol. 87, pt. 1, pp. 77-86, 2004.

[5] A. Itou, H. Ebara, H. Nakajima, K. Wada, and O. Hashimoto, "An experimental study of a wave absorber using a frequency-selective surface," Microw. Opt. Technol. Lett., vol. 28, pp. 321-323, 2001.

[6] G. I. Kiani, A. R. Weily, and K. P. Esselle, "A novel absorb/transmit FSS for secure indoor wireless networks with reduced multipath fading," IEEE Microw. Wireless Compon. Lett., vol. 16, no. 6, pp. 378-380, 2006.

[7] B. A. Munk, P. Munk, and J. Pryor, "On designing Jaumann and circuit analog absorbers for oblique angle of incidence," IEEE Trans. Antennas Propag., vol. 55, no. 1, Jan. 2007.

[8] A. P. Raiva, F. J. Harackiewicz, and J. Lindsey, "Frequency selective surfaces: Design of broadband elements and new frequency stabilization techniques," presented at the Antenna Application Symp., Monticello, Ill, Sep. 17-19, 2003. 